\title{
Periodic variability in the emission spectrum of T Tauri
}

\author{
N. Z. Ismailov ${ }^{1,2}$, N. K. Quliev ${ }^{1}$, O. V. Khalilov ${ }^{1}$, and W. Herbst ${ }^{3}$ \\ 1 Shamakha Astrophysical Observatory, National Academy Sciences of Azerbaijan, Shamakha, Azerbaijan \\ e-mail: wherbst@wesleyan.edu \\ 2 Baku State University, 23 Z. Khalilov St. 370148 Baku, Azerbaijan \\ 3 Astronomy Department, Wesleyan University, Middletown, CT 06419, USA
}

Received 23 October 2009 / Accepted 15 December 2009

\begin{abstract}
Context. Long timescale periodic variations in T Tauri stars may be related to changes in their protoplanetary disks. Aims. A lengthy homogeneous series of spectral observations of the CTTS T TauN has been analyzed. Methods. We search for periodicity in the equivalent widths of the emission lines in the optical and in the UV. Results. Significant periodic variation is found in the equivalent widths of $\mathrm{H} \beta$ and CaII emission features on a time scale of $33 \pm$ 1.5 days. Variations in the UV emission lines based on archival IUE spectra are also consistent with this period. Conclusions. This is clearly not the rotation period of the star, which is known to be 2.8 days, and must arise somewhere in the magnetosphere, disk or outflow region.
\end{abstract}

Key words. stars: pre-main sequence - stars: variables: general

\section{Introduction}

Binary and multiple systems are common among T Tauri stars (Duguennoy \& Mayor 1991; Mathieu et al. 2000), many having been discovered by the techniques of speckle interferometry, lunar occultation and adaptive optics in the infrared (Dyck et al. 1982; Simon et al. 1995; Kohler \& Leinert 1998). For the prototype of T Tauri stars, T Tau itself, one finds three components, labeled $\mathrm{T}$ TauN, $\mathrm{T}$ TauSa and $\mathrm{T}$ TauSb, with angular separations of $0.68^{\prime \prime}$ between $\mathrm{N}$ and $\mathrm{S}$ and $0.12^{\prime \prime}$ between $\mathrm{Sa}$ and Sb (Koresco 2000; Duchene et al. 2002). A large number of observations is required to solve the orbits of such resolved systems. Spectroscopic monitoring over long time scales comparable to the orbital period can be very interesting. An example of such a study is provided by Koehler et al. (2008) who report preliminary spectroscopic data for the $\mathrm{T} \mathrm{Tau} \mathrm{Sa} / \mathrm{Sb}$ pair with a period of $21.66 \mathrm{yr}$ and masses of $2.1 \pm 0.2 M_{\odot}$ and $0.8 \pm 0.1 M_{\odot}$ for $\mathrm{Sa}$ and $\mathrm{Sb}$ respectively. While at the present time there are insufficient astrometric observations to confidently construct the full orbit, various authors have constructed partial solutions with periods ranging from 21 to 33 years and very similar masses for the components (Tamazian 2004; Duchene et al. 2006).

Despite these studies, it is still not yet clear whether the multiplicity of many $\mathrm{T}$ Tauri stars can be linked to the ubiquitous short and long term brightness and spectral variations observed for them. The well known periodic variation of $\mathrm{T}$ TauN with a period of 2.8 days, which was discovered by Herbst et al. (1986) is undoubtedly due to the axial rotation of the star, as those authors and Zajtseva et al. (1988) discuss. A longer term variation in the visual brightness of this object with a period of 6 years was reported by Ismailov \& Samus (2003). This result was confirmed by Mel'nikov \& Grankin (2005). All of the work on $\mathrm{T}$ TauN searching for periodicity to date has been based on broad band $(U B V)$ photometry. At these wavelengths, there is no substantial contribution to the total light of the system from the $\mathrm{T}$ TauS components. Therefore, Ismailov et al. (2009) argue that the long term cyclic variations in brightness must come from $\mathrm{T}$ TauN and not from $\mathrm{T}$ TauS.

$\mathrm{T}$ Tauri stars commonly show short term periodic variations on time scales of 2-10 days which are generally interpreted as arising from the axial rotation of a star with an inhomogeneous surface, having either hot or cool spots (Herbst et al. 1987, 1994). When variations are larger in the $U$ band than in the $R$ and $I$ bands it is often indicative of the presence of hot, as opposed to cool, spots (Herbst et al. 1994; Bouvier et al. 1995). The spectroscopic signature of these hot, accretion spots is "veiling" of the absorption lines by a hot continuum (Valenti et al. 1993; Gulbring et al. 1998). It is supposed that the hot spots form on the surface of the star as a result of an accretion shock (Lynden-Bell \& Pringle 1974; Königl 1991; Shu et al. 1994). In his theoretical work on this subject Lamzin $(1995,1998)$ describes the structure of such impact zones within the magnetospheric accretion model.

Clearly, when there is variable accretion and/or flare activity on the star due to magnetic reconnections, it may be difficult to find the underlying stable period that represents its axial rotation rate. The magnetospheric accretion model predicts that there will be correlations between UV and optical radiation. Such correlations have been reported for BP Tau (Simon et al. 1990) and RU Lup (Giovanelli et al. 1990), for example. In the case of flare activity on young stars it is also possible to find a correlation between the UV and optical radiation components (Montmerle et al. 1993). Clearly a large amount of observational work is required to test and refine the magnetospheric accretion model, given the range and complexity of the phenomena involved. Long term monitoring at a variety of wavelengths and including spectral and photometric programs is required. 
Table 1. IUE spectra of T Tau included in this study.

\begin{tabular}{lclc}
\hline \hline Spectrum No & JD & Spectrum No & JD \\
\hline SWP03172LL & 2443809.31654 & LWR15028LL & 2445344.68961 \\
SWP07006LL & 2444539.47296 & LWR15040LL & 2445346.17693 \\
SWP10543LL & 2444548.06631 & LWP02061LL & 2445623.90535 \\
SWP10600LL & 2444557.37234 & LWP02076LL & 2445625.93758 \\
SWP10613LL & 2444560.55512 & LWP02084LL & 2445626.97263 \\
SWP49799LL & 2449361.91497 & LWP02090LL & 2445627.80559 \\
SWP49804LL & 2449362.89171 & LWP06932LL & 2446355.96905 \\
LWR04224LS & 2443973.44117 & LWP06933LL & 2446355.99975 \\
LWR04224LL & 2443973.45936 & LWP06934LL & 2446356.02895 \\
LWR04248LS & 2443975.51465 & LWP06940LL & 2446356.94328 \\
LWR04250LL & 2443975.59042 & LWP06941LL & 2446356.97546 \\
LWR05943LS & 2444173.45613 & LWP06942LL & 2446357.00884 \\
LWR05943LL & 2444173.46271 & LWP02094LL & 2446358.94947 \\
LWR05944LL & 2444173.60462 & LWP27199LL & 2449361.95988 \\
LWR09296LL & 2444557.73207 & LWP27200LL & 2449362.08508 \\
LWR09297LL & 2444557.79424 & LWP27207LL & 2449362.92781 \\
LWR15027LL & 2445344.55742 & LWP27208LL & 2449363.02192 \\
\hline
\end{tabular}

For UV studies the final archive spectra of the IUE satellite is an excellent resource for research into the behavior of young stars. Several atlases of spectral lines in the wavelength range 1175-3000 ^ for young stars have been constructed (Valenti et al. 2000; Johns-Krull et al. 2000; Valenti et al. 2003). These are excellent resources for time domain studies of the variability of T Tau in the UV. In this paper we make use of a series of optical spectra of T Tau obtained from 1971-1988 on which the equivalent widths of the $\mathrm{H} \beta$ line as well as the $\mathrm{H}$ and $\mathrm{K}$ lines of CaII have been measured. In addition, we have studied the time variation of several UV lines using spectra from the IUE archive. Our main goal has been to search for any periodic variations that may be present in the spectrum of $\mathrm{T}$ Tau, complementing the studies already done on photometry.

\section{Observations}

\subsection{Optical spectra}

Spectroscopy of T Tau was carried from 1971 until 1988 at the Cassegrain focus of the $2 \mathrm{~m}$ telescope of Shamakha Astrophysical Observaotry in Azerbaijan. The instrument is a $2 \times 2$ prism "Canberra" spectrograph with inverse dispersion of $94 \AA / \mathrm{mm}$ at $\mathrm{H} \gamma$. The specral range covered was from 3600 to $5100 \AA$ and the spectral resolution is $\sim 4 \AA$ at $\mathrm{H} \gamma$. A full description of the spectral material and details of measurements of emission equivalent widths has been presented in Table 1 of the paper by Ismailov et al. (2006). We chose to focus on the $\mathrm{H} \beta$ and CaII lines for this time series study because we have many more measurements of their equivalent widths than for any of the other (weaker) lines. Standard errors on the equivalent width measurements are typically $15 \%$ or less for both $\mathrm{H} \beta$ and the $\mathrm{H}$ and $\mathrm{K}$ lines. In total, we have 167 spectrograms on which these lines could be measured. An earlier study of the emission spectrum of T Tau based on these same spectra indicated that there was a possibly periodic variation in the equivalent widths on a time scale of 16-18 days, attributed to flare activity of the star (Guliyev 1991).

Nightly averages of the equivalent widths were used for the period search, resulting in a set containing 42 points spread over the 18 year interval of the observations. We use the Scargle (1982) periodogram method as recommended by Horne $\&$ Baliunas (1986) to search for periods. The computer code was from Antokhin et al. (1995) and in this paper one can also find a detailed description of the technique and its application. The method allows one to calculate the false alarm probability, $F$, for any peak in the periodogram of height, $z$. This is the probability that a peak of height $z$ will occur by chance in a data set of random noise. The probability that the data actually contain a particular periodic signal is thus $1-F$. If the normalized power spectrum is calculated at $N_{i}$ independent frequencies then $F$ is given by the following relationship (Horne \& Baliunas 1986):

$F=1-\left(1-\mathrm{e}^{-z}\right)^{N_{i}}$

The frequency interval in which it is valid to search for a period is roughly $1 / T<v<1 / 2 \Delta t_{\min }$ where $\Delta t_{\min }$ is the minimum time interval between adjacent observations.

\subsection{UV Spectra}

\subsubsection{Standard stars}

As is well known, the IUE archives contain spectrograms that have been obtained with three different cameras. The Short Wavelength Prime (SWP) camera covers the spectral range 1175 to $2000 \AA$ while the Long Wavelength Prime (LWP) and Long Wavelength Redundant (LWR) cameras cover the range of 2000-3000 A. The spectral resolution is about $6 \AA$. After standard processing (Nichols et al. 1994) each spectrum is provided in a gif format. Images of the UV spectra of T Tau are readily available through the information base SIMBAD. It is clear from a casual inspection that there are numerous overlapping lines creating blends and complicating analysis.

Because of the heterogeneity of the spectra and to account for interstellar reddening we have applied a classical method of processing spectrograms, The spectra are normalized to the local continuum intensity $\left(I_{0}\right)$ and the residual intensity, $R_{\lambda}=1-I / I_{0}$, and half width, $\Delta_{1 / 2}$, defined as the full width at half maximum (FWHM), determined. For emission lines, $I$ is the absolute intensity at the peak of the line and $I_{0}$ is the continuum level of the line. In such measurements, there is an unavoidable uncertainty due to the problem of setting the spectral continuum level. This was done carefully by eye using the Paint tool in Windows XP operating system.

To check the reliability of this process, we chose about 40 standard dwarf stars (class V luminosity class) for the IUE 
Table 2. List of measured lines in the UV spectrum of T Tau.

\begin{tabular}{cccc}
\hline \hline Wavelength $(\AA)$ & Element & Wavelength (A) & Element \\
\hline 1154 & O II (PW) & 1697 & Si I (297)a \\
1238 & N V (107) & 1808 & Si II (292) \\
1255 & H2 R(3) 1-3, R(5) 1-3 & 1817 & Si II (292) \\
1270 & H2 P(5) 1-3, R(0) 1-3 & 1833 & Si I (237)a \\
1296 & Si III (320), S I (K1), & 1891 & Si III] \\
1304 & O I (215), Si II (292) & 1908 & C III] \\
1335 & C II (211), H2 R(0) 0-4, R(1) 1-4 & 1933 & Fe I (56) \\
1355 & C I (211) & 1940 & Fe I (56)a \\
1393 & P(1) 0-5, Si IV (319) & 1957 & Fe I (56)a \\
1402 & H2 P(2) 0-6, Si IV (319) & 2330 & C II] \\
1433 & H2 R(4) 1-6, R(3) 1-6, & 2338 & Fe II (56) \\
1450 & H2 P(6) 1-6, P(10) 0-5, & 2354 & Fe II (56) \\
1467 & C I (211) & 2392 & Fe II (56) \\
1486 & H2 R(3) 1-7 & 2469 & Fe II (56 \\
1503 & H2 R(6) 1-7, P(6) 1-7 & 2514 & Fe II (56) \\
1519 & H2 P(3) 0-7, S II (P2) & 2631 & Fe II (56) \\
1548 & C IV (211), H2 P(3) 1-8 & 2672 & Cr II (1) \\
1561 & C I (211), H2 R(6) 1-8 & 2743 & Fe II (56) \\
1580 & Fe II (105) & 2761 & Fe II (56) \\
1587 & O II, O III ? & 2800 & Mg II (RII) \\
1602 & C I, S II (P2) & 2844 & Fe I (56) \\
1618 & Fe II (105) & 2929 & Fe I (56) \\
1639 & Fe II (105), He II & 2973 & Fe I (56) \\
1665 & O III] & 3137 & Si II (292) \\
1683 & Si I (237)a & 3214 & Fe I (56) \\
\hline
\end{tabular}

Low Dispersion Catalogue (LDC). The range of spectral classes covered was from B0 to M0. Using our procedure we measured the equivalent widths of various lines suitable to the spectral class. In the interval of B0-A5 these were, for SWP spectra: Ly $\alpha$

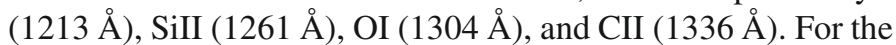
LWP spectra in the spectral type range G0 to K7, we chose FeII (2607 $\AA$ and $2746 \AA$ ), the MgII doublet (2800 $)$, CrII (2866 $⿱$ ) , FeI/CuI (3097 ̊̊). Our measured central residual intensities were plotted as a function of the spectral class of the stars and showed a monotonic relationship. Based on the scatter about the mean relationship we estimate that our determination of this quantity is accurate to better than $10 \%$. The estimated errors on $\Delta_{1 / 2}$ is, of course, larger and can be up to $15-20 \%$. We conclude on the basis of this exercise that the IUE spectrograms are quite suitable for carrying out both spectral classification and the type of spectral analysis we employ here.

\subsubsection{T Tau}

In Table 1 we list all of the available IUE spectra of T Tau that were suitable for processing and use in this study. Seven of these are of type SWP and 27 are of type LWP or LWR. All selected SWP spectra are of good quality and we measured spectral parameters successfully. The LWP and LWR spectra are not so uniformly good. In some, the level of the continuum and the signalto-noise ratio is low and we have not been able to measure all of the lines in every case.

Table 2 is a list of all of the spectral lines that we have been able to identify and measure in the T Tau UV spectra. The symbol "a" is used to designate clean absorption lines. As may be seen, there are flourescent $\mathrm{H}_{2}$ lines visible in the SWP spectra. These have been discussed already by several authors (Valenti et al. 2000; Johns-Krull et al. 2000; Valenti et al. 2003; Herczeg et al. 2006)
For each spectrogram, a residual intensity, $R_{\lambda}$ in units of the local continuum intensity, and halfwidth, $\Delta_{1 / 2}$, have been measured for each line. Measurements have shown variability in some lines. In Tables 3 and 4 we present values of $R_{\lambda}$ for all relevant lines in the different wavelength regions, respectively. We have also computed emission equivalent widths based on our measured values of $\Delta_{1 / 2}$ and the assumption of a triangular line profile. However, the errors in equivalent width measures are relatively large and, for an analysis of the variability, we have limited ourselves to $R_{\lambda}$. The analysis has shown that the unblended lines of $\mathrm{H}_{2}$ do not vary significantly. On the other hand, spectral emission lines of other elements do show significant variation and, as we show in the next section, are consistent with periodicity.

\section{Results}

The equivalent widths measured on the optical spectra were analyzed for periodicity using the Scargle method as described. Figure 1 shows the power spectrum of the data in the upper panels and of the window function in the lower panels. On the left is $\mathrm{H}_{\beta}$ and on the right is the averaged data for the $\mathrm{H}$ and $\mathrm{K}$ lines of CaII. In both cases the highest peak in the power spectrum rises to or above the level required for a $\sim 90 \%$ confidence level that it is not a false alarm. Nearly identical results are found for $\mathrm{H}_{\beta}$ and for each of the CaII lines. The observed maximum for the power spectrum always lies in the frequency range of 0.0300 to $0.0350 \mathrm{~d}^{-1}$, corresponding to a period between 29.85 and $33.33 \mathrm{~d}$. We, therefore, conclude that there is a significant periodicity in these data with a period of $33 \pm 1.5 \mathrm{~d}$. In Fig. 2 we show a plot of the data phased with a period of $33 \mathrm{~d}$ and a coherent variation is apparent in all three lines. An ephemeris giving the times of minimum equivalent width is Min $=$ JD2440989.143+33E. 
Table 3. Intensities, $\left|R_{\lambda}\right|$ on SWP spectra of T Tau.

\begin{tabular}{ccccccc}
\hline \hline No. & Spectrum No & JD & 1296SiII & 1304 OI & $1335 \mathrm{H} 2$ & 1394SiIV.H2 \\
\hline 1 & SWP03172LL & 2443809.31654 & 5.1 & 7.8 & 5.2 & 5.6 \\
2 & SWP07006LL & 2444539.47296 & 4.1 & 9.9 & 3.9 & 3.7 \\
3 & SWP10543LL & 2444548.06631 & 2.4 & 5.4 & 2.2 & 2.7 \\
4 & SWP10600LL & 2444557.37234 & 2.3 & 1.9 & 5.6 & 2.0 \\
5 & SWP10613LL & 2444560.55512 & 3.1 & 7.9 & 1.5 & 2.6 \\
6 & SWP49799LL & 2449361.91497 & 3.1 & 5.4 & 2.0 & 3.9 \\
7 & SWP49804LL & 2449362.89171 & 3.0 & 5.7 & 2.6 & 3.6 \\
\hline No. & 1403SiIV & $1433 \mathrm{H} 2$ & $1450 \mathrm{H} 2$ & $1486 \mathrm{H} 2$ & $1500 \mathrm{H} 2$ & $1546 \mathrm{CIV}$ \\
1 & 4.5 & 4.1 & 4.3 & 3.6 & 4.7 & 14.2 \\
2 & 3.3 & 2.6 & 3.8 & 3.8 & 4.2 & 12.9 \\
3 & 2.5 & 2.8 & 3.9 & 3.9 & 4.7 & 8.2 \\
4 & 3.4 & 3.8 & 3.1 & 3.7 & 4.5 & 5.2 \\
5 & 2.5 & 3.9 & 3.6 & 3.7 & 5.0 & 7.5 \\
6 & 3.5 & 3.0 & 4.7 & 4.2 & 4.6 & 10.6 \\
7 & 4.0 & 3.4 & 4.9 & 3.8 & 4.5 & 13.5 \\
\hline No. & $1640 \mathrm{HeII}$ & $1808 \mathrm{SiII}]$ & $1817 \mathrm{SiII}]$ & $1891 \mathrm{SiIII}]$ & $1908 \mathrm{CIII}]$ & \\
1 & 4.5 & 2.6 & 4.6 & 2.7 & 3.3 & \\
2 & 4.3 & 3.2 & 5.7 & 2.3 & 2.4 & \\
3 & 2.9 & 1.4 & 3.8 & 1.9 & 2.6 & \\
4 & 12.9 & 4.1 & 2.2 & 4.4 & 1.6 & \\
5 & 3.5 & 1.2 & 3.4 & 1.3 & 2.9 & \\
6 & 4.4 & 2.6 & 5.2 & 2.1 & 3.6 & \\
7 & 7.9 & 3.6 & 7.4 & 3.3 & 4.7 & \\
\hline
\end{tabular}

Table 4. Intensities, $\left|R_{\lambda}\right|$, for selected lines on LWP and LWR spectra of T Tau.

\begin{tabular}{|c|c|c|c|c|c|c|c|}
\hline Spectr & JD & Phase $(P=33 \mathrm{~d})$ & $2336 \mathrm{CII}]$ & 2630FeII & $2743 \mathrm{FeII}$ & $2800 \mathrm{MgII}$ & $2975 \mathrm{FeII}$ \\
\hline LWR04224LS & 2443973.44117 & 0.4332 & 11.6 & 4.2 & 3.2 & 23.9 & 2.6 \\
\hline LWR04224LL & 2443973.45936 & 0.4338 & 10.7 & 3.9 & & 19.4 & 1.2 \\
\hline LWR04248LS & 2443975.51465 & 0.4961 & & & & 40.2 & \\
\hline LWR04250LL & 2443975.59042 & 0.4984 & 15.3 & 3.9 & 2.0 & 6.5 & 0.8 \\
\hline LWR05943LS & 2444173.45613 & 0.4943 & & 2.8 & & 27.8 & \\
\hline LWR05943LL & 2444173.46271 & 0.4945 & 3.3 & 3.4 & 1.9 & 23.3 & 3.0 \\
\hline LWR05944LL & 2444173.60462 & 0.4988 & 7.9 & 3.5 & 2.5 & 4.1 & 1.1 \\
\hline LWR09296LL & 2444557.73207 & 0.1390 & 11.0 & 3.1 & 1.6 & 11.5 & 1.2 \\
\hline LWR09297LL & 2444557.79424 & 0.1409 & & & & 38.1 & \\
\hline LWR15027LL & 2445344.55742 & 0.9823 & 13.3 & 3.0 & 1.3 & 3.5 & 0.8 \\
\hline LWR15028LL & 2445344.68961 & 0.9863 & 9.5 & 5.5 & 2.4 & 15.6 & \\
\hline LWR15040LL & 2445346.17693 & 0.1313 & 4.8 & 2.6 & & 33.9 & \\
\hline LWP02061LL & 2445623.90535 & 0.4473 & 19.1 & 7.8 & 4.6 & 56.8 & 2.6 \\
\hline LWP02076LL & 2445625.93758 & 0.5089 & 7.3 & 7.7 & 4.0 & 55 & 3.0 \\
\hline LWP02084LL & 2445626.97263 & 0.5403 & & & & 20.6 & \\
\hline LWP02090LL & 2445627.80559 & 0.5655 & & & & 33.4 & \\
\hline LWP06932LL & 2446355.96905 & 0.6311 & & & & 37 & \\
\hline LWP06933LL & 2446355.99975 & 0.6320 & 18.3 & 4.9 & & 37.9 & \\
\hline LWP06934LL & 2446356.02895 & 0.6329 & 7.6 & 3.7 & & 30.6 & \\
\hline LWP06940LL & 2446356.94328 & 0.6606 & 7.4 & 1.4 & 2.6 & 33.8 & 2.0 \\
\hline LWP06941LL & 2446356.97546 & 0.6616 & 7.0 & 2.8 & 1.9 & 17.3 & 1.0 \\
\hline LWP06942LL & 2446357.00884 & 0.6626 & 6.8 & 2.2 & 1.7 & 16.4 & \\
\hline LWP02094LL & 2446358.94947 & 0.7214 & & & & 22.3 & \\
\hline LWP27199LL & 2449361.95988 & 0.7217 & 9.0 & 3.8 & & 12.9 & \\
\hline LWP27200LL & 2449362.08508 & 0.7255 & 17.7 & 6.3 & & 33.0 & \\
\hline LWP27207LL & 2449362.92781 & 0.7510 & 5.5 & 2.8 & 1.7 & 8.1 & 0.8 \\
\hline LWP27208LL & 2449363.02192 & 0.7539 & & 4.3 & & 28.0 & \\
\hline
\end{tabular}

While there are not enough data in the UV to warrant a separate periodogram analysis, it is possible to phase these data with the same ephemeris as found for the optical emission lines. Figure 3 displays such data for the UV emission lines of OI (1304 ̊), SiIII (1296 ̊), SiIV (1395 ̊) and CIV (1546 ̊). It is evident that most of the SWP range spectral lines show a 33 day periodic variation in intensity. In Fig. 4, we show the phased intensity measurements for the following lines: HeII (1640 $\AA$ ), SiII] (1817 $\AA$ ), CIII] (1908 $\AA$ ) and $\mathrm{H}_{2}$ (1486 and $1500 \AA$ ). Again, except for $\mathrm{H}_{2}$ which does not show significant variation, the data appear to follow the 33 d periodicity. Finally, in Fig. 5, we show the phased data for the following lines in the LWP and LWR spectra: FeII (2743 and $2975 \AA$ ), the MgII doublet (2800 $⿱$ ) and CII] (2336 ̊). The FeII lines show a significant variation linked 

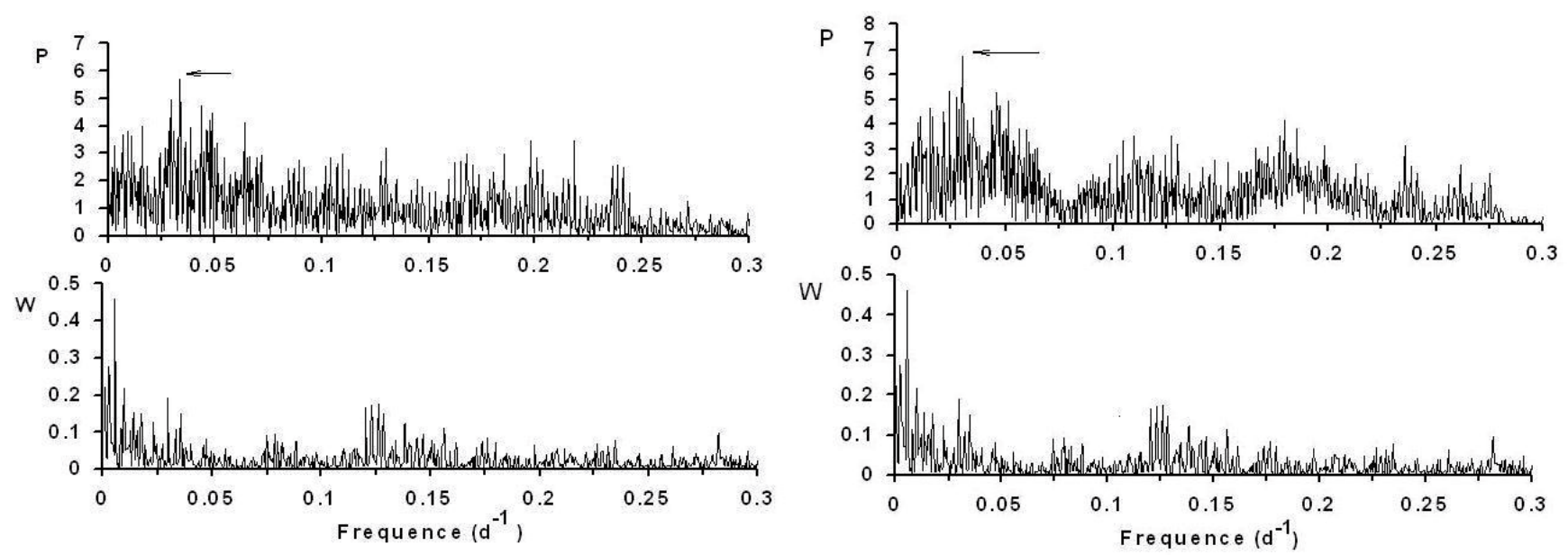

Fig. 1. Power spectrum and window parameters for equivalent widths of emission lines of $\mathrm{H} \beta($ left $)$ and $\mathrm{H}$ and $\mathrm{K}$ lines of CaII (right).

to the reported ephemeris although some data points (3 or 4) do not agree. In MgII and CII] we do not see much agreement with the phase, although there does seem to be a maximum of intensity at about phase $=0.5$.

We conclude from this analysis that there is a significant variation of spectral emission lines in the optical and UV on a time scale of about 33 days and that this variation is periodic. The lines systematically increase in strength for 15 to 18 days and then decline in intensity over the remainder of the cycle. Obviously this cyclic behavior must have persisted over a very long period of time since our data set spans 18 years.

\section{Discussion}

From our spectroscopic data we can estimate the influence of the emission line variations on the photometric brightness of the star within the spectral range of 3600 to $5100 \AA$. One may write that

$\Delta m=2.5 \log \frac{I_{0}+\frac{\Sigma W_{\lambda}}{\Delta \lambda}}{I_{0}}$

where $\Delta m$ is the magnitude of the variability of the brightness due to the emission lines, $I_{0}$ is the intensity of the continuum, and $\Sigma W_{\lambda}$ is the summation of the equivalent widths of all the emission lines within the spectral range $\Delta \lambda$. In our case, $\Delta \lambda=1400 \AA$ and $\Sigma W_{\lambda}=\sim 15 \AA$ during weak emission phases and $\sim 60 \AA$ during strong emission phases (Ismailov et al. 2006). Accordingly, the emission lines will contribute about 0.012 mag to the broad band photometric brightness during weak emission phases and about 0.045 mag during active conditions. We note that a seasonal variation in the mean brightness of $\mathrm{T}$ TauN of this amplitude is typical.

The photometric amplitude of T Tau is typically $0.05 \mathrm{mag}$ in $B$ and $V$ and 0.038 in $R$ and 0.022 in $I$ (Herbst et al. 1986; Zajtseva 1989). According to Herbst et al. (1986) the periodicity of $2.8 \mathrm{~d}$ is not seen in the UV or in the $\mathrm{H} \alpha$ line. It is very likely that the $2.8 \mathrm{~d}$ period arises from the axial rotation of a star with an inhomogeneous surface brightness distribution on its photosphere. It is not clear what relationship, if any, the $33 \mathrm{~d}$ period in the emission lines reported here has to the $2.8 \mathrm{~d}$ period in the broadband optical photometry. It is interesting, however, that the emission line variability can apparently change the brightness of the star in U and B, at least, by about the same amount measured for the axial rotation variation.
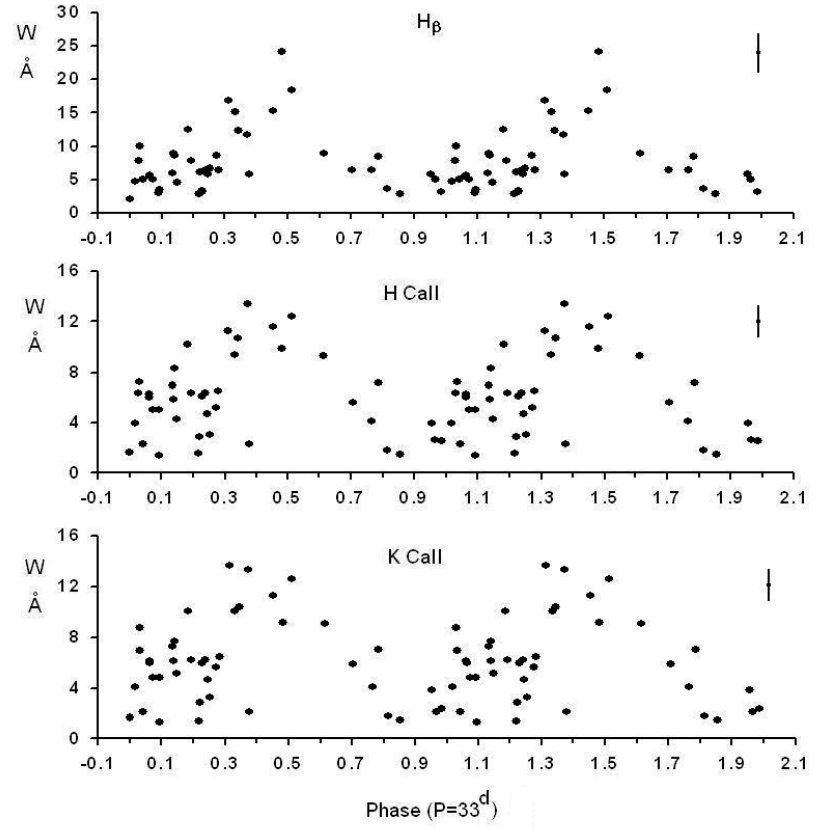

Fig. 2. Phased curves of equivalent widths of the emission lines of HI and CaII for a period of $33 \mathrm{~d}$.

In spite of the fact that the emission line variability reported here may affect the photometric measurements of the star in $\mathrm{U}$ and $\mathrm{B}$, there is no hint of the 33 day periodicity in the power spectrum of the star as reported by Herbst et al. (1986). There are several possible explanations for this fact. First, the power spectrum reported there applies to $V$ magnitude measurements, which were most numerous, not $B$ and $U$ measurements, which are most affected by the emission lines. Second, the cadence of the observations in that study was optimized for short period detection, not periods as long as a month. Typically, individual observing runs on the star did not extend for times as long as a month or two, as required for clear detection of the period reported here. Finally, it may be that the longer time scale periodicity found here may be more ephemeral than the axial rotation period of the star. It may simply not have been present (or as 

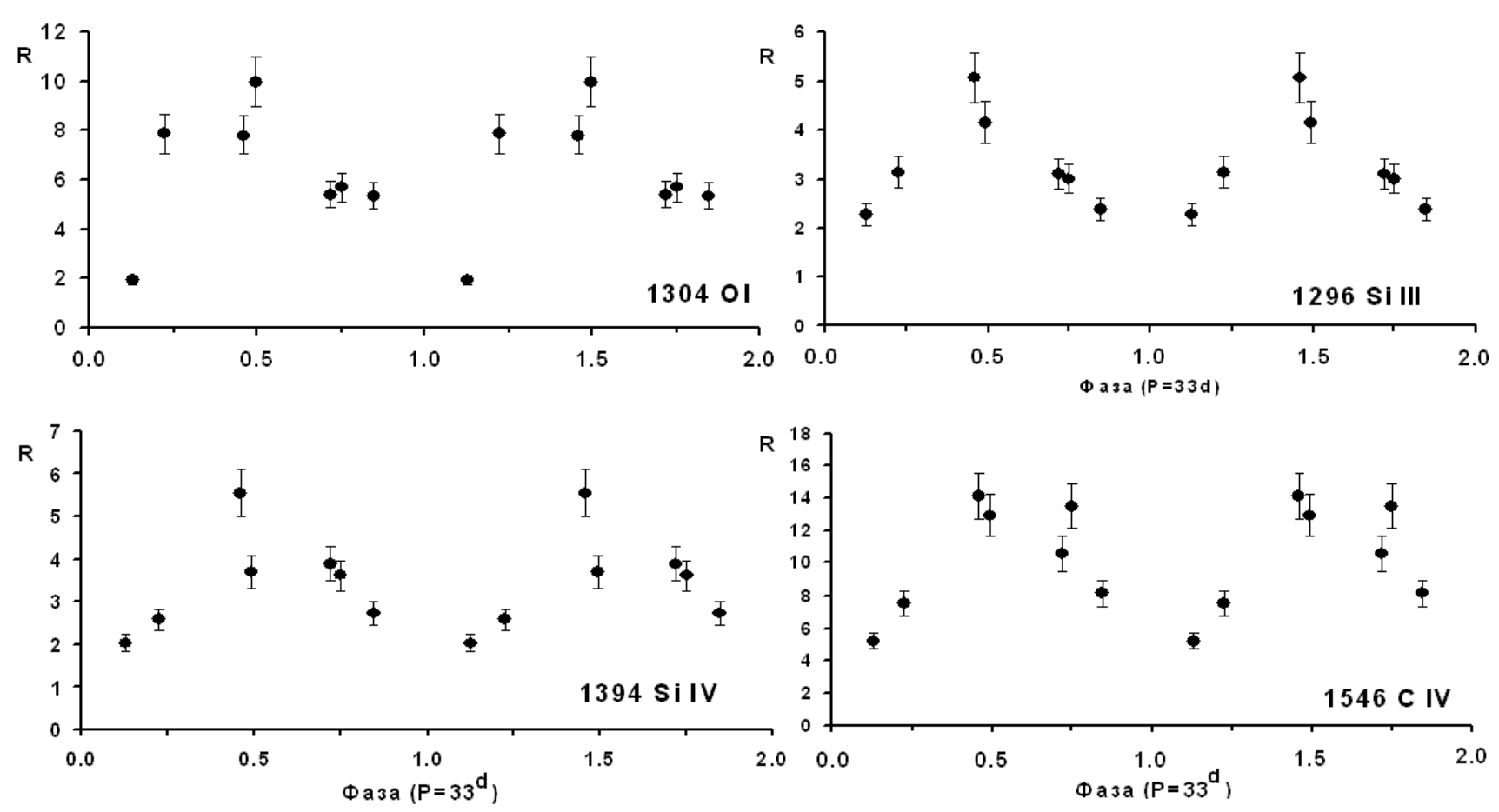

Fig. 3. Phased curves of the residual intensity of several UV spectra lines for a period of $33 \mathrm{~d}$.
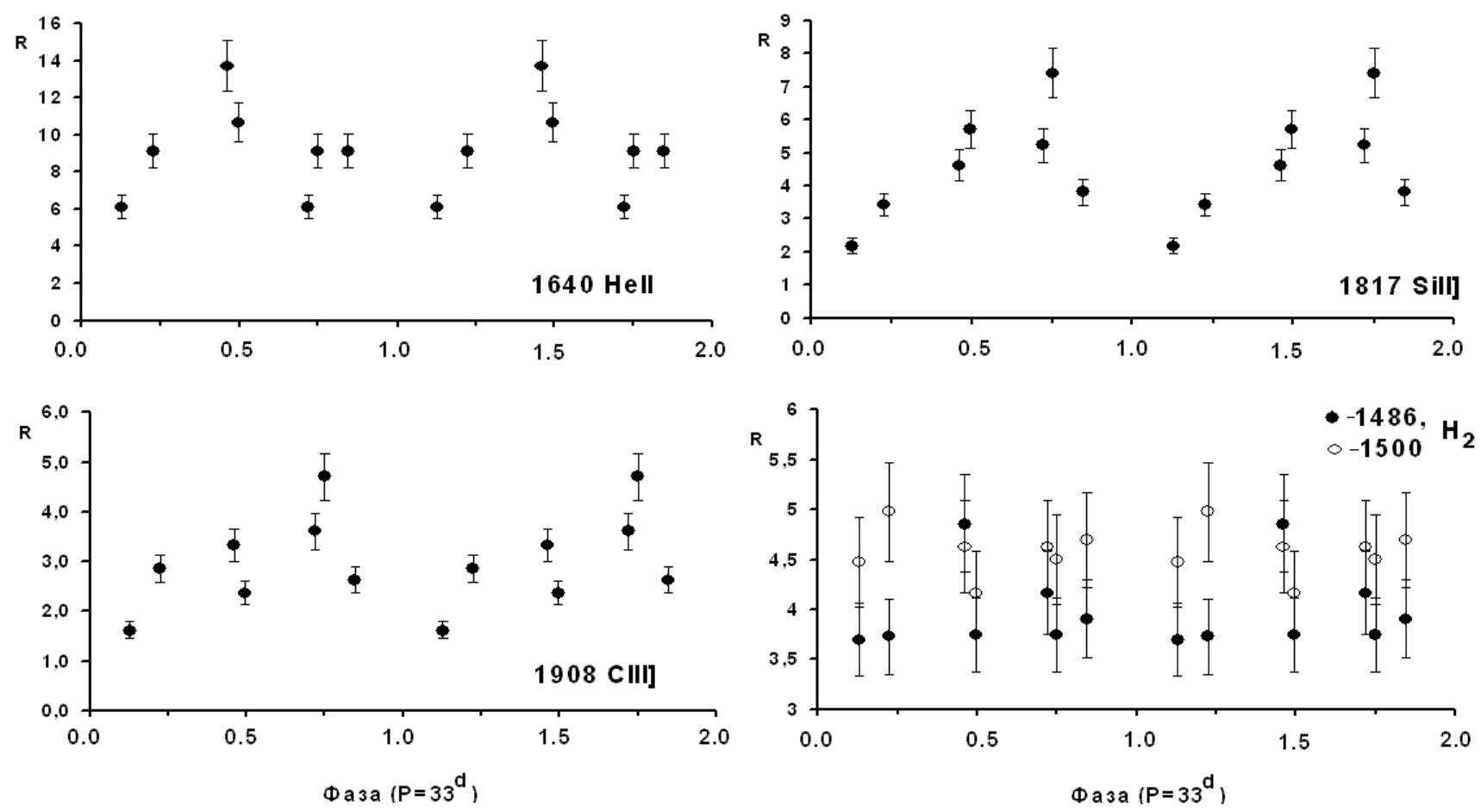

Fig. 4. Phased curves of the residual intensity of several UV spectra lines for a period of $33 \mathrm{~d}$.

strongly present) during the epoch of the photometry thus far analyzed.

If the 33 day periodicity arises in the disk, then it will be located at about $0.57 \mathrm{AU}$ from the star, assuming a mass of $2.1 \mathrm{M}_{\odot}$ and Keplerian motion. Since the source region of the UV and optical emission lines measured here is more likely well within the magnetosphere and/or in the jet region, it is hard to understand how a periodicity as long as $33 \mathrm{~d}$ could arise. It appears, based on the photometric period and the measured $v \sin i$ that we view T TauN close to pole-on (Herbst et al. 1986). Perhaps some rotation effect along the axis of the jet is involved and it may even be a clue to the details of the jet launching mechanism. This, 
N. Z. Ismailov et al.: Periodic variability in the emission spectrum of T Tauri
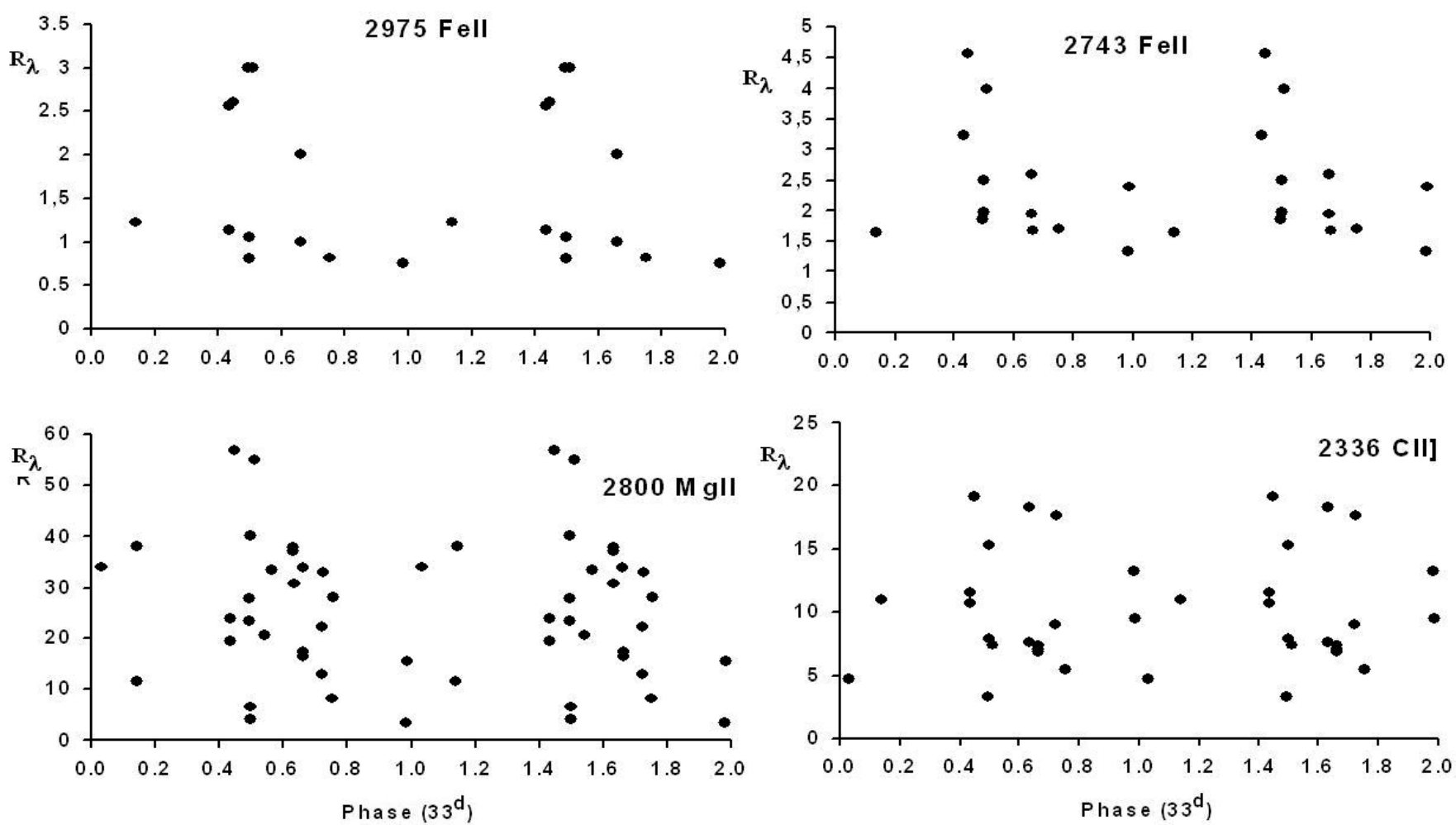

Fig. 5. Phased curves of the residual intensity of several UV spectra lines for a period of $33 \mathrm{~d}$.

however, is beyond the scope of the present investigation to elucidate further. Clearly, we require additional data and monitoring, both spectroscopically and photometrically and, hopefully including UV monitoring, to confirm the reported period and further our understanding of this complex system.

Acknowledgements. One of us (W.H.) would like to thank NASA through its Origins program for partial support of this work.

\section{References}

Antokhin, I., Bertrand, J.-F., Lamontagne, R., et al. 1995, AJ, 109, 817 Bertout, C., Basri, G., \& Bouvier, J. 1988, ApJ, 330, 350

Bouvier, J., Cavino, E., Kovo, O., et al. 1995, A\&A, 299, 89

Duchene, G., Ghez, A. M., \& McCabe, C. 2002, ApJ, 568, 771

Duchene, G., Beust, H., Adjali, F., Konopacky, Q. M., \& Ghez, A. M. 2006, A\&A, 457, L9

Duguennoy, M., \& Mayor, M. 1991, A\&A, 248, 485

Dyck, H. M., Simon, T., \& Zuckerman, B. 1982, ApJ, 255, L103

Giovanelli, L., Rossi, C., Errico, L., et al. 1990, in Evalution in Astrophysics, ed.

E. J. Rolfe, ESA Publications, ESTEC, Noordwijk, ESA SP-310, 231

Gomez de Castro, A. I., \& Fernandez, M. 1996, MNRAS, 283, 55

Gullbring, E., Hartmann, L., Brice, C., et al. 1998, ApJ, 492, 323

Guliev, N. Kh. 1991, Kinematika i Fizika Nebesnykh Tel, 7, 5

Hartmann, L., Hewett, R., Stahler, S., et al. 1986, ApJ, 309, 275

Herbst, W., Booth, J. F., Chugainov, P. F., et al. 1986, ApJ, 310, 71

Herbst, W., Booth, J. F., Korett, D. L., et al. 1987, AJ, 94, 137

Herbst, W., Herbst, D. K., Grossman, E. J., et al. 1994, AJ, 108, 4810

Herczeg, G. J., Linsky, J. L., Walter, F. M., Gahm, G. F., \& Johns-Krull, C. M. 2006, ApJS, 165, 256

Horne, J. H., \& Balinas, S. L. 1986, ApJ, 302, 757
Ismailov, N. Z., \& Samus, N. N. 2003, IBVS, 5382

Ismailov, N. Z., Quliev, N. Kh., Alieva, A. A., et al. 2006, v.26, No 5

Ismailov, N. Z., Shustarev, P. N., Alieva, A. A., \& Alimardanova, F. N. 2009, Astron. Rep. 53(7), 643

Johns-Krull, C. M., Valenti, J. A., \& Linsky, J. I. 2000, ApJ, 539, 815

Koehler, R., Ratzka, T., Herbst, T. M., et al. 2008, A\&A, 482, 929

Kohler, R., \& Leinert, C. 1998, A\&A, 331, 977

Königl, A. 1991, ApJ370, L39

Koresco, C. D. 2000, ApJ, 531, L147

Lamzin, S. A. 1995, A\&A, 295, L20

Lamzin, S. A. 1998, Astron. Rep., 42, 322

Lynden-Bell, D., \& Pringle, J. E. 1974, MNRAS, 168, 603

Mathieu, R. D., Ghez, A. M., Jensen, E. L. N., et al. 2000, in Protostars and Planets. IV, ed. V. Mannings, A. P. Boss, \& S. S. Ressel (Tuscon: Univ. of Arizona Press), 703

Melnikov, S. Yu., \& Grankin, K. M. 2005, Astron. Lett., 31, 109

Montmerle, T., Feigelson, E. D., Bouvier, J., et al. 1993, in Protostars and Planets III, ed. E. H. Levy, \& I. J. Lunine (Tuscon: University of Arizona press), 689

Nichols, J. S., Garhart, M. P., De La Pena, M. D., et al. 1994, NASA IUE Newsletter, 53

Petrov, P. P. 2003, Astrophys., 46, 611

Scargle, J. D. 1982, ApJ, 263, 835

Shu, F. N., Najita, J., Ostriker, E., et al. 1994, ApJ, 429, 781

Simon, M., Ghez, A. M., Leinert, Ch., et al. 1995, ApJ, 443, 625

Simon, T., Vrba, F. J., \& Herbst, W. 1990, AJ, 100, 1957

Tamazian, V. S. 2004, AJ, 127, 2378

Valenti, J. A., Basri, G., \& Johns, C. M. 1993, AJ, 10, 2024

Valenti, J. A., Johns-Krull, C. M., \& Linsky, J. I. 2000, ApJS, 129, 399

Valenti, J. A., Fallon, A. A., \& Johns-Krull, C. M. 2003, ApJS, 147, 305

Zajtseva, G. V. 1989, Astrophys., 313, 489

Zajtseva, G. V., Tarasov, K. V., \& Chernova, G. P. 1988, Soviet Astron. Lett. 14(4), 259 